\title{
LA DISTRIBUCIÓN DE COMPETENCIAS COMO LÍMITE A LA EFECTIVA PROTECCIÓN DE LA SALUD (SSTC 134/2017, DE 16 DE NOVIEMBRE; 140/2017, DE 30 DE NOVIEMBRE, Y 145/2017, DE 14 DE DICIEMBRE)
}

\author{
Juan Antonio Maldonado Molina \\ Profesor Titular de Derecho \\ del Trabajo y de la Seguridad Social \\ Facultad de Derecho \\ Universidad de Granada \\ jmaldo@ugr.es
}

\section{INTRODUCCIÓN}

La llamada «descentralización territorial del Estado del bienestar», fenómeno polémico y complejo en un escenario geopolítico como el de nuestro país, ha tenido numerosas manifestaciones en las últimas décadas más allá del campo de la asistencia social y los servicios sociales, que era su ámbito tradicional, pasando por las pensiones, las rentas mínimas de inserción y la asistencia sanitaria ${ }^{1}$. En este contexto han sido frecuentes los choques entre las pretensiones autonómicas de legislar sobre determinadas materias y la posición del Gobierno central que las consideraba «legislación básica», lo que las excluiría, en consecuencia, del ámbito autonómico. Existe ya una prolija jurisprudencia constitucional en la materia, que no procede recordar, en la que se han sentado los límites del diálogo entre normas estatales y autonómicas, que podría condensarse en la idea de que la norma básica actúa como un mínimo común mejorable por la normativa autonómica. Sin embargo, esta doctrina parece haber quebrado con las

${ }^{1}$ Sobre tales cuestiones nos remitimos a nuestro estudio J. A. Maldonado Molina, «Distribución de competencias en materia de seguridad social y asistencia social: Sentencia TC 239/2002, de 11 de diciembre», en J. GarCÍA MuRCIA (dir.), El control de constitucionalidad de las normas laborales y de Seguridad Social. 20 casos de jurisprudencia constitucional, Pamplona, Aranzadi, 2015, pp. 687-720. 
sentencias objeto de comentario. En diciembre de 2017 se han dictado tres pronunciamientos del Tribunal Constitucional (en adelante, TC) que tienen en común el mismo detonante: una reacción autonómica frente a una previa reforma restrictiva estatal en el ámbito sanitario. $\mathrm{Y}$ ante ese desarrollo normativo con el que algunas Comunidades ${ }^{2}$ han tratado de restituir los derechos previos a la reforma, el Gobierno presenta conflicto positivo de competencia, que ha sido resuelto en los tres casos en sentido favorable al mismo. En todos los supuestos, el TC se ha limitado a analizar la distribución competencial, sin entrar a valorar si el derecho material a la asistencia sanitaria se veía o no conculcado. Pero la gran novedad reside en la interpretación dada a qué debe considerarse como legislación básica, al impedir desarrollos autonómicos que mejoren el mínimo estatal si lo que se quiere mejorar es la titularidad del derecho, diferenciando, en consecuencia, el ámbito subjetivo (que no sería mejorable) respecto del objetivo (que sí es mejorable).

\section{LAS NORMAS AUTONÓMICAS CUESTIONADAS Y LOS ARGUMENTOS DEL ABOGADO DEL ESTADO}

La crónica de los hitos normativos que desembocan en las sentencias comentadas es el que sigue. Basado competencialmente en el art. 149.1.16 $\mathrm{CE}^{3}$, el Real Decreto-ley 16/2012, de 20 de abril, sobre medidas urgentes para garantizar la sostenibilidad del Sistema Nacional de Salud y mejorar la calidad y seguridad de sus prestaciones, aprobó tres medidas principalmente: redefine la condición de asegurado (dejando sin cobertura sanitaria a determinados colectivos $)^{4}$, modifica la cartera de servicios y amplía el copago farmacéutico. En definitiva, se aprueban unas medidas que -en palabras del propio Tribunal Constitucional— suponen «un giro en la anterior

2 País Vasco (STC 134/2017, de 16 de noviembre), Navarra (STC 140/2017, de 30 de noviembre) y Comunidad Valenciana (STC 145/2017, de 14 de diciembre).

${ }^{3}$ Que, como es sabido, atribuye al Estado la competencia exclusiva en materia de bases y coordinación general de la sanidad y sobre la legislación de productos farmacéuticos. Esta competencia subsume, por ser más específica, la genérica del art. 149.1.1 (conforme a la doctrina del TC recogida en la STC 136/2012).

${ }^{4}$ En particular, la cobertura de los extranjeros ya no se condiciona a que estén empadronados, sino a que estén registrados o autorizados como residentes en España (salvo determinados supuestos previstos en el art. 3 ter de la Ley 16/2003). Norma desarrollada reglamentariamente por Real Decreto 1192/2012, de 3 de agosto, que regula la condición de asegurado y de beneficiario a efectos de la asistencia sanitaria en España con cargo a fondos públicos a través del Sistema Nacional de Salud. 
política de progresiva extensión de la asistencia sanitaria gratuita o bonificada a partir de la creación del Sistema Nacional de Salud» ${ }^{5}$.

Sentada la constitucionalidad de este giro ${ }^{6}$, lo cierto es que parecía resituarse el espacio entre el mínimo garantizado por el Estado y sus eventuales mejoras autonómicas, dado que el mínimo estatal deja de ser universal. Con ese presupuesto, algunas Comunidades pronto aprobaron normas en las que extendían la cobertura por encima del mínimo garantizado por el Estado, con fundamento general en el principio de autonomía y fundamento específico en la competencia autonómica de desarrollo legislativo en materia de sanidad. Así:

- País Vasco: Decreto 114/2012, de 26 de junio, sobre régimen de las prestaciones sanitarias del Sistema Nacional de Salud en el ámbito de la Comunidad Autónoma de Euskadi, que tras permitir en su apartado primero el acceso a las prestaciones de la cartera de servicios del Sistema Vasco de Salud a los asegurados o beneficiarios del Sistema Nacional de Salud, añade en sus apartados segundo y tercero nuevos supuestos de adquisición del derecho de acceso a las prestaciones sanitarias respecto a los previstos en la legislación estatal.

- Navarra: Ley Foral 18/2012, de 19 de octubre, sobre la complementación de las prestaciones farmacéuticas en la Comunidad Foral de Navarra, que actúa en el ámbito de la financiación de los medicamentos, recogiendo como titulares del derecho a la complementación de las prestaciones farmacéuticas a personas que, con arreglo a la nueva normativa básica estatal, no ostentan la condición de asegurado o beneficiario; Decreto Foral 117/2012, de 31 de octubre, por el que se modifica el Decreto Foral 640/1996, de 18 de noviembre, que establece el procedimiento y las condiciones para el acceso a las prestaciones del régimen de universalización de la asistencia sanitaria pública en la Comunidad Foral de Navarra, y Ley Foral 8/2013, de 25 de febrero, por la que se reconoce a las personas residentes en Navarra el derecho de acceso a la asistencia sanitaria gratuita del sistema público sanitario de Navarra.

- Comunidad Valenciana: Decreto Ley 3/2015, de 24 de julio, que regula el acceso universal a la atención sanitaria en la Comunidad Valenciana.

5 FJ 8 de la STC 139/2016, citada expresamente en el FJ 5 de la STC 134/2017.

${ }^{6}$ SSTC 139/2016, de 21 de julio; 33/2017, de 1 de marzo; 63/2017, de 25 de mayo; 64/2017, de 25 de mayo; 97/2017, de 17 de julio, y 98/2017, de 20 de julio. 
Frente a estas normas, el Gobierno presenta conflictos positivos de competencia que el abogado del Estado sustenta en tres títulos competenciales:

- El art. 149.1.2 CE, que reserva al Estado la competencia exclusiva en materia de extranjería. Al amparo de este título competencial se dictó la Ley Orgánica 4/2000, de 11 de enero, sobre derechos y libertades de los extranjeros en España y su integración social, cuyo art. 12 remite en bloque el reconocimiento y regulación del derecho a la asistencia sanitaria de los extranjeros a la regulación vigente en materia sanitaria. La norma impugnada, al establecer un régimen diferente al desarrollado por el Estado, está vulnerando las competencias estatales en la materia.

- El art. 149.1.16 CE, que declara la competencia exclusiva de bases y coordinación general de la sanidad atribuida al Estado.

- El art. 149.1.17 CE, en tanto que estima que la asistencia sanitaria se incluye dentro de lo que se denomina «asistencia social interna» al sistema de la Seguridad Social y, desde este punto de vista, la competencia del Estado queda amparada en el título del régimen económico de la Seguridad Social.

De forma resumida, la argumentación se condensa en que se apoya en la jurisprudencia constitucional ${ }^{7}$ que declara que la determinación de los beneficiarios de las prestaciones sanitarias constituye una de las claves del sistema, de manera que la fijación de dichos beneficiarios por la norma básica no es sólo el mínimo común normativo exigible en todo el territorio español para garantizar la igualdad de todos los españoles en el acceso a las prestaciones del Sistema Nacional de Salud (art. 149.1.16 CE), sino una condición básica para todos los ciudadanos en el ejercicio de un derecho constitucional (art. 149.1.1 CE) como es el derecho a la protección de la salud reconocido en el art. 43.1 CE.

Siguiendo esta línea argumental, el abogado del Estado señala que «la legislación estatal distingue claramente el ámbito subjetivo de la asistencia sanitaria pública del ámbito objetivo», de tal suerte que «en el subjetivo, como elemento primario y nuclear para garantizar la igualdad en las condiciones de ejercicio del derecho a la salud, el Estado ejerce una competencia exclusiva ex art. 149.1.1 CE no susceptible de desarrollo. Los titulares de la asistencia sanitaria pública son los que son. Iguales en todo el Sistema Nacional de Salud. Por el contrario, en el ámbito objetivo es donde las

\footnotetext{
7 STC 136/2012, de 19 de junio.
} 
Comunidades pueden mejorar el mínimo prestacional que establecen las bases estatales, introduciendo técnicas, tecnologías o procedimientos adicionales a los que pueden afectar recursos financieros adicionales» (antecedente 2 de la STC 134/2017).

\section{LOS ARGUMENTOS DEL CONSTITUCIONAL}

El TC sigue una misma línea argumental en las tres sentencias referidas al considerar que presentan un problema constitucional similar, sin perjuicio de que en algún caso se plantearan otras cuestiones que finalmente no llegaron a ser dilucidas dado que, al estimarse la invalidez de la norma impugnada por ser contraria al orden constitucional de competencias, quedaron sin resolverse ${ }^{8}$.

Así, y centrándonos en el tema competencial, el TC sostiene que a la hora de delimitar quién tenga derecho a la prestación sanitaria, las diferentes Comunidades Autónomas han «de adecuar necesariamente sus regulaciones a los conceptos de asegurado y de beneficiario en la forma y con los límites configuradores que establece aquella normativa estatal básica, de tal manera que no puedan extender el ámbito subjetivo de la relación prestacional más allá de los límites que configura el art. 3 de la Ley 16/2003, en la redacción operada por el art. 1.Uno del Real Decreto-ley 16/2012» (FJ 2 del STC 145/2017).

En su FJ 5, la STC 134/2017 afirma que «(e)s la primera vez que se plantea ante este Tribunal de un modo directo la cuestión de la naturaleza de la regulación básica del elemento subjetivo del Sistema de Salud». Indica que «lo que ahora procede es determinar si la normativa básica estatal colma en su integridad el ámbito subjetivo del derecho de acceso a las prestaciones sanitarias, de tal manera que en el Sistema Nacional de Salud establecido para todo el territorio del Estado el derecho de acceso a la cartera de prestaciones sanitarias tenga el mismo nivel de cobertura subjetiva». Y considera «como formal y materialmente básico el conjunto de preceptos que delimitan el concepto de quiénes hayan de tener acceso al catálogo de prestaciones del Sistema Nacional de Salud».

8 Así, en la sentencia relativa a la norma de la Comunidad Valenciana (STC 145/2017, de 14 de diciembre) también se planteó en el recurso del Gobierno que el Consell de la Generalitat Valenciana ejerció su potestad legislativa de urgencia sin respetar los requisitos que, de acuerdo con lo dispuesto en los arts. 86 CE y 44.2 del Estatuto de Autonomía, deben respetar los decretos-leyes. 
De este modo, se acogen los argumentos de la Abogacía del Estado en el sentido de que la mejora autonómica no puede tener lugar en el ámbito subjetivo del derecho, ya que ese ámbito en realidad constituye el núcleo básico de regulación que garantiza la igualdad a nivel estatal.

En consecuencia, la normativa básica estatal cierra toda posibilidad a las normas autonómicas de desarrollo para configurar un sistema de acceso a las prestaciones sanitarias que no atienda a los conceptos de asegurado o de beneficiario. Y como resultado de tal interpretación es inconstitucional una norma autonómica que extienda el acceso a las prestaciones sanitarias a las personas extranjeras no registradas ni autorizadas como residentes en España y con residencia efectiva que no tengan la condición de aseguradas ni de beneficiarias del Sistema Nacional de Salud.

Pero no se queda la sentencia en considerar inconstitucionales las mejoras autonómicas que versen sobre el ámbito subjetivo, sino que abre la posibilidad de vetar mejoras si éstas no respetan los límites que las bases del Estado fijen. De este modo, no sólo hay un marco básico, sino unos límites infranqueables que, como ahora desarrollaremos, son los principios informadores que inspiran al Gobierno en la regulación de una institución.

En efecto, en esta segunda línea argumental, el TC parte de que «el derecho a la salud recogido en el art. $43 \mathrm{CE}$ es un mandato que ha quedado constitucionalmente enunciado como principio rector y no como derecho fundamental, y, por tanto, es el legislador quien ha de determinar las técnicas apropiadas para llevar a cabo su plasmación». Aunque reconoce que no significa que estemos ante normas meramente programáticas, asume que «tales principios son decisiones axiológicas jurídico-objetivas que determinan que, a la hora de interpretar el modelo de distribución de competencias» (FJ 5 de la STC 134/2017) y que «por imperativo del criterio de unidad de la Constitución», se les deba dotar de la mayor fuerza normativa posible, y ello se traduce, en el caso de la sanidad, en la capacidad del Estado de preservar la existencia de un sistema normativo sanitario nacional «con una regulación uniforme mínima y de vigencia en todo el territorio español» que garantice la igualdad de atención. Pues bien, indica el TC que «el reconocimiento a las Comunidades Autónomas de desarrollar para mejorar el ámbito prestacional fijado por el Estado se halla en función, precisamente, de los límites y del alcance que las propias bases del Estado fijen, con fundamento en los intereses generales que aquél justifique». 
Considera que con el Real Decreto-ley 16/2012 hubo un cambio de modelo, un giro?. Y los principios axiológicos que inspiran ese nuevo modelo deben ser tenidos en cuenta a la hora de analizar si se respetan o no las bases estatales. Así, señala el TC que «(t)al cambio en la configuración de las bases, que el preámbulo del Real Decreto-ley justifica en la necesidad de imponer "una clarificación armonizada de la condición de asegurado, a efectos de la prestación de los servicios sanitarios y socio-sanitarios" financiada con fondos públicos, es lo que determina que, a la hora de delimitar, conforme al mencionado sistema de bases, quién tenga derecho a la prestación sanitaria, las diferentes Comunidades Autónomas, en cuanto administraciones sanitarias que tienen a su cargo el desarrollo normativo y la función ejecutiva de la prestación sanitaria, hayan de adecuar necesariamente sus regulaciones a los conceptos de asegurado y de beneficiario en la forma y con los límites configuradores que establece aquella normativa estatal básica, de tal manera que no puedan extender el ámbito subjetivo de la relación prestacional más allá de los límites que configura el art. 3 de la Ley 16/2003, en la redacción operada por el art. 1.1 del Real Decreto-ley 16/2012».

Es una línea argumental que, como vemos, el TC reserva para el ámbito subjetivo, pero entendemos que también podría dar pie al ámbito objetivo de la prestación sanitaria, cerrando la puerta a que se complementara autonómicamente la intensidad y/o la extensión objetiva de la atención sanitaria.

Sea como fuere, el TC reconduce esa interpretación a las mejoras del ámbito subjetivo, señalando que «la normativa básica estatal cierra toda posibilidad a las normas autonómicas de desarrollo para configurar un sistema de acceso a las prestaciones sanitarias que no atienda a los conceptos de asegurado o de beneficiario que han establecido las bases recogidas en el tantas veces citado art. 3 de la Ley 16/2003».

\footnotetext{
9 Se remite en este punto a la STC 139/2016, que en su FJ 8 señaló que el RDL 16/2012 «supone un giro en la anterior política de progresiva extensión de la asistencia sanitaria gratuita o bonificada a partir de la creación del Sistema Nacional de Salud», toda vez que los criterios de delimitación de la titularidad del derecho de acceso a las prestaciones sanitarias ahora seleccionados son diferentes a los que establecía la anterior redacción del art. 3 de la Ley 16/2003, que atendía a otros parámetros distintos como eran el de la residencia del titular en territorio español.
} 


\section{LOS VOTOS PARTICULARES}

En las tres sentencias se acompañan dos votos particulares (en las Sentencias 140/2017 y 145/2017 se remiten a los formulados en la STC 134/2017): el primero suscrito por cuatro magistrados ${ }^{10} \mathrm{y}$ el segundo por uno ${ }^{11}$.

En el primero se disiente del fallo al considerar que el mínimo estatal (antes universal) «resulta ahora susceptible de mejora por las Comunidades Autónomas, con fundamento general en el principio de autonomía y fundamento específico en la competencia autonómica de desarrollo legislativo en materia de sanidad», de modo que consideran que es plenamente constitucional que una Comunidad desarrolle legislativamente tales mínimos. Afirman que «(e)s esta una concepción consustancial a la diversidad territorial que otorga a nuestro ordenamiento una estructura compuesta, que supone necesariamente una diversidad de regímenes jurídicos, pues la autonomía significa precisamente la capacidad de adoptar políticas propias en el marco de la Constitución y del Estatuto».

Apoyan sus argumentos en una consolidada jurisprudencia constitucional que no introduce distingos en la noción de legislación básica, limitándose a considerarla como un mínimo común denominador establecido que puede mejorarse frente al fallo de la sentencia, que excluye del mínimo al ámbito subjetivo del Derecho.

Por tanto, consideran que esa interpretación es claramente rupturista con la que se ha venido manteniendo. Literalmente señalan: «(E)xpresamos nuestro rotundo desacuerdo con esa desmedida noción de lo básico, que no se diferencia sustancialmente de una competencia legislativa exclusiva del Estado. Se socava por esta vía una más que consolidada doctrina constitucional sobre las competencias normativas compartidas en un Estado de estructura plural».

Avanzando en esta idea, recuerdan que «lo que está vedado al legislador autonómico es precisamente lo contrario: no puede empeorar el régimen de financiación de la prestación farmacéutica haciéndolo más gravoso para el usuario» ${ }^{12}$, de modo que «negar a las Comunidades Autónomas

${ }^{10}$ Don Fernando Valdés Dal-Ré, don Juan Antonio Xiol Ríos, don Cándido CondePumpido Tourón y doña María Luisa Balaguer Callejón.

${ }^{11}$ Don Antonio Narváez Rodríguez.

12 Recuerdan que esto «quedó advertido en las SSTC 71/2014 y 85/2014, que anularon las tasas autonómicas conocidas como "euro por receta" de las Comunidades Autónomas de Cataluña y Madrid». 
su capacidad de mejorar la posición de los usuarios en la financiación de la prestación farmacéutica se opone a lo hasta ahora sostenido por el Tribunal y, en definitiva, les impide el ejercicio de sus competencias de desarrollo legislativo en materia de sanidad».

Pero, además, cuestionan que la constitucionalidad de una norma pueda valorarse sólo en términos estrictamente competenciales, considerando que «(l)a efectividad del derecho a la protección de la salud hubiera debido presidir la interpretación de los títulos competenciales confrontados a fin de respaldar, de entre las diversas interpretaciones posibles, aquellas que razonablemente permitan cumplir el mandato proclamado en el art. 43 CE y alcanzar sus objetivos». Entienden que ésta es «una idea que ha sido esbozada en la jurisprudencia constitucional, pero que lamentablemente todavía no ha cobrado la necesaria relevancia en su generalizada aplicación. La Constitución sólo puede ser comprendida e interpretada correctamente cuando se la entiende como unidad» ${ }^{13}$.

Todo ello conduce a que en ese voto particular se recoja un alegato a favor de la defensa del derecho material a la salud como seña de identidad del Estado del bienestar. Así, es especialmente elocuente el siguiente párrafo: «A resultas de esta involución normativa, ese legislador excepcional que es el Gobierno ha colocado entre paréntesis el fatigoso pero constante proceso de construcción y consolidación en la sociedad española del Estado de bienestar. Por este lado, la concepción del Welfare State, propia del constitucionalismo social más avanzado, ha sido suplantada, en aras al logro de presuntos objetivos tales como la eficiencia y la racionalización económicas, por la del Workfare State».

Tras ese alegato, el primer voto particular llama la atención de que la realidad ha demostrado que «como reacción a este injustificado viraje y con la abierta finalidad de evitar las situaciones de desprotección, con riesgos evidentes para la salud no sólo individual, sino también colectiva, generadas por el Real Decreto-ley 16/2012 en relación con los colectivos sociales más vulnerables en una economía de mercado progresivamente globalizada, la práctica totalidad de las Comunidades Autónomas ha procedido, en el curso de los últimos años, a adoptar medidas de retorno al principio de universalidad de la asistencia sanitaria». Pues bien, denuncia el voto que son las Comunidades Autónomas que de forma abierta y directa han abordado el problema (vía intervención normativa) las que ahora se

${ }^{13}$ Se remiten a las SSTC 227/1988, de 29 de diciembre, FJ 13; 239/2002, de 11 de diciembre, FJ 3; 30/2011, de 16 de marzo, FJ 5, y 233/2015, de 5 de noviembre, FJ 2. 
encuentran con el litigio planteado por el Gobierno central, mientras que otras (la mayoría de ellas) «eligieron opciones políticas extremas y camufladas de carácter indirecto e informal, al estilo del dictado de instrucciones a los centros sanitarios de su competencia de que faciliten a todos los ciudadanos, con criterios de gratuidad y al margen de su situación jurídica o de su nacionalidad, las prestaciones sanitarias precisas para garantizar su derecho a la salud», lo cual desde luego no conduce a garantizar ni la igualdad de todos los ciudadanos españoles ni garantiza el principio de seguridad jurídica.

El segundo voto particular es el formulado por Antonio Narváez Rodríguez, que se da la circunstancia de que a la vez le correspondió ser el ponente de la sentencia a la que formula el voto discrepante.

Discrepa parcialmente, porque sólo comparte la declaración de inconstitucionalidad del art. 2 de la norma vasca, que prevé nuevos supuestos de adquisición del derecho de acceso a las prestaciones sanitarias respecto a los previstos en la legislación estatal, pero sin que tal inconstitucionalidad debiera haber afectado a la capacidad autonómica de ampliar el ámbito subjetivo de las prestaciones sanitarias. Indica el magistrado que: «La razón no es otra que la de considerar que una doctrina, que se encuentra ya consolidada en el ámbito objetivo de las prestaciones sanitarias, tendría también que haber sido aplicada por este Tribunal al mencionado ámbito subjetivo de las prestaciones». Concluye indicando que: «El planteamiento del régimen de distribución de competencias que, sobre esta concreta cuestión y en materia de atención sanitaria, hace la sentencia no se corresponde con la doctrina ampliamente reiterada por este Tribunal respecto de la dinámica constitucional bases-desarrollo que establece el art. 149.1.16 CE, al tiempo que, en mi opinión, se ha dejado pasar una nueva oportunidad de elaborar una construcción de lo básico que devuelva algún sentido a la distribución de competencias sobre la indicada materia, amén de no haber podido avanzar en la construcción de una interpretación integradora de la Constitución como un todo».

\section{CONCLUSIONES}

1. En 2016, el TC estimó constitucional el Real Decreto-ley 16/2012, de 20 de abril, sobre medidas urgentes para garantizar la sostenibilidad del Sistema Nacional de Salud y mejorar la calidad y seguridad de sus prestaciones, admitiendo —eso sí- que el legislador (el ejecutivo, más bien) 
dio «un giro en la anterior política de progresiva extensión de la asistencia sanitaria gratuita o bonificada a partir de la creación del Sistema Nacional de Salud».

En estas sentencias de final de 2017 es el TC el que da el giro, distinguiendo por primera vez los elementos subjetivos de los objetivos en la determinación de la noción de «legislación básica» e impidiendo desarrollos autonómicos que mejoren el mínimo estatal si lo que se quiere mejorar es la titularidad del derecho, diferenciando, en consecuencia, el ámbito subjetivo (que no sería mejorable) respecto del objetivo (que sí lo sería).

2. La línea argumental que usa el TC se centra en vetar la mejora del ámbito subjetivo, pero entendemos que también podría dar pie a limitar mejoras autonómicas en el ámbito objetivo de la prestación sanitaria. Y es que, como literalmente señala, «el reconocimiento a las Comunidades Autónomas de desarrollar para mejorar el ámbito prestacional fijado por el Estado se halla en función, precisamente, de los límites y del alcance que las propias bases del Estado fijen, con fundamento en los intereses generales que aquél justifique». Para el TC, los principios rectores (concretados en la norma por el legislativo) «son decisiones axiológicas jurídico-objetivas que determinan que, a la hora de interpretar el modelo de distribución de competencias, se les deba dotar de la mayor fuerza normativa posible», determinando hasta dónde pueden llegar las mejoras autonómicas. El límite ya no será el mínimo estatal, sino qué buscaba el legislador con ese mínimo común denominador.

3. Esta interpretación, en última instancia, lo que hace es supeditar la efectiva promoción del derecho a la salud a los intereses generales, pero considerando que los intereses generales son los que el legislador estatal estime convenientes en cada momento. Así, un marco normativo autonómico que lograra implementar de forma efectiva el art. 43 de la Constitución correría el riesgo de devenir inconstitucional si la mejora que hiciese de la normativa básica fuera contradictoria con las directrices políticas del Gobierno central, lo que, además de vaciar de contenido al Estado autonómico, vacía de sentido la declaración de Estado social y democrático de Derecho, ya que primaría la distribución competencial al máximo desarrollo de los derechos sociales. 